Complexe, écologique, créative : La ville contemporaine et la transformation sociale

Mario Salomone et Marcella Messina

\author{
(2) OpenEdition \\ Journals \\ Édition électronique \\ URL : http://journals.openedition.org/ere/3269 \\ DOI : $10.4000 /$ ere.3269 \\ ISSN : 2561-2271 \\ Éditeur \\ Centr'ERE
}

Référence électronique

Mario Salomone et Marcella Messina, "Complexe, écologique, créative : La ville contemporaine et la transformation sociale », Éducation relative à l'environnement [En ligne], Volume 7 | 2008, mis en ligne le 20 septembre 2008, consulté le 21 février 2020. URL : http://journals.openedition.org/ere/3269 ; DOI : $10.4000 /$ ere.3269

Ce document a été généré automatiquement le 21 février 2020. 


\title{
Complexe, écologique, créative : La ville contemporaine et la transformation sociale
}

\author{
Mario Salomone et Marcella Messina
}

1 Le thème de l'environnement urbain est encore trop peu abordé par la recherche en éducation relative à l'environnement (ERE). Or plus de la moitié de la population mondiale vit désormais dans les villes et ce taux dépasse $80 \%$ en Europe. Il importe de développer une citoyenneté plus consciente et plus active, une société plus participative et démocratique, dont le sens de la responsabilité et l'action collective s'appuient sur une éthique plus profonde de notre rapport à la Nature et aux autres humains, conduisant à l'émergence de systèmes socio-économiques et de modèles de production et de consommation plus justes et plus écologiques. Il nous faut repenser la ville et établir avec elle un rapport différent et « réconcilié ».

2 Or la ville se présente souvent (notamment dans les grandes agglomérations des pays en développement, mais aussi dans grand nombre de métropoles du monde industrialisé) comme un monstre énergivore. La ville contemporaine est de plus en plus déchirée par des contrastes et des contradictions socio-économiques. Par ailleurs, elle est marquée par de graves problèmes environnementaux (pollutions diverses, génération importante de déchets, trafic, etc.) et par de vastes zones de dégradation. Elle est saisie par des transformations profondes. Il existe à ce sujet toute une littérature sociologique (par exemple, Cesareo, 2001 ; Mazzette, 2003 ; Mela, 2006).

Peut-on échapper à ce destin? Les personnes et les collectivités peuvent-elles suivre une voie différente afin que, grâce à leur rôle actif et critique, les villes deviennent des communautés conscientes, des lieux de citoyenneté active et de pleine expression des droits humains, civils et sociaux? Peut-on envisager les villes comme des lieux où il fait bon vivre et où la vie est saine?

Dans cette brève analyse, nous tenterons d'illustrer deux voies, distinctes mais étroitement liées, la pensée critique et la créativité, comme des stratégies possibles et 
appropriées pour construire et développer des communautés urbaines plus solidaires et participatives contribuant à de nouvelles dynamiques au sein de nos métropoles.

\section{La ville, épicentre des transformations}

5 Dans les villes « globales » (les villes « mondialisées »), comme l'évoque Bauman (2005), l'épicentre des transformations ne se situe souvent qu'au sein des grandes aires urbaines selon une logique de réseau international dont les nœuds sont les quartiers métropolitains où se concentrent les fonctions les plus avancées du capitalisme. Les villes deviennent donc, entre autres, l'objet de nouveaux et d'intenses flux de population ainsi que d'une profonde redistribution des revenus. Il y a une dichotomie de plus en plus évidente entre les quartiers constitués d'une élite de la mondialisation hautement professionnalisée et les quartiers où s'entasse une population très nombreuse, défavorisée.

6 L'effet de ce mouvement est déchirant : tandis que certains quartiers sont surqualifiés et qu'ils font l'objet d'investissements urbains considérables, d'autres zones tombent en proie à la dégradation et deviennent marginales. Dans certaines villes d'Afrique et d'ailleurs, c'est le centre qui est pauvre et la périphérie qui est riche. Dans beaucoup de cas, les quartiers plus riches sont conçus comme des citadelles exclusives, complètement autonomes et indépendantes du centre urbain et des autres quartiers des villes desquelles ils font partie.

7 On peut se référer à la ville comme lieu où émerge une nouvelle question sociale : "face aux tensions qui se créent entre les flux de population et les lieux, entre les fonctions et les populations, entre les intérêts et les cultures, la ville devient l'objet le plus utile pour interpréter la transformation contemporaine » (traduction libre, Caritas italiana, 2007). Fragmentation, désagrégation, perte de l'enracinement territorial, marginalité, dégradation, exclusion sociale, telles sont quelques-unes des caractéristiques des villes d'aujourd'hui (Sassen, 2003). Il est même possible de relever à l'œil nu l'augmentation des iniquités et de l'hétérogénéité culturelle de la population : chaque année, 60 à 70 millions de personnes viennent grossir les zones urbaines et métropolitaines du monde (UNFPA, 2007 ; UNDESA, 2008a, 2008b).

8 Du point de vue relationnel, les habitants de la ville se rencontrent dans le cadre de rapports très fractionnés et parcellisés. Les contacts entre les personnes peuvent avoir lieu face à face, mais ils sont malgré tout bien souvent impersonnels, superficiels, transitoires. La réserve et l'indifférence manifestées par les habitants des villes dans leurs rapports peuvent être considérées comme un expédient pour se défendre contre les demandes et les attentes des autres. Bien que les liens traditionnels soient affaiblis, voire même effrités, le quotidien urbain comporte aussi une dimension beaucoup plus vaste d'interdépendances entre les personnes ainsi qu'une forme plus complexe, fragile et impalpable de relations réciproques. Voilà pourquoi le sociologue Richard Sennett (2006) décrit la ville comme un établissement humain où il est possible que des individus, étrangers les uns aux autres, se rencontrent, sans pour autant donner lieu à un rapport constructif, actif et durable.

9 Bien que de nombreuses années se soient écoulées depuis l'époque où Louis Wirth (1998, édition originale 1938) brossait, avec une capacité d'analyse extraordinaire, un tableau des réalités sociales urbaines, ses propos sont plus que jamais actuels. Du point de vue des liens sociaux, d'après Wirth (1998), l'ampleur de l'urbanisation a généré des 
situations dépersonnalisantes et d'anonymat. Par ailleurs, l'affaiblissement des liens de parenté, le déclin de la signification sociale de la famille et la diminution des liens de voisinage ont entraîné la désagrégation de la base traditionnelle de solidarité sociale (Wirth, 1998).

\section{Iniquités croissantes}

10 Réduites à des «lieux de conception, de production et d'organisation de désirs individuels pratiqués par groupes et alimentés par le marché et par ses règles" (traduction libre, Mazzette, 2006, p. 14), les villes de la «société liquide » c'est-à-dire celles issues de la modernité «liquide " (selon Bauman, 2002) caractérisée par la liberté, la flexibilité, mais aussi par l'insécurité, la fragilité et l'impalpabilité des liens entre les humains, semblent être frappées par des problèmes communs : absence d'un plan unitaire d'organisation du territoire, diminution d'espaces publics, déclin de socialisation, décalage croissant entre les habitants et les institutions avec la crise conséquente de la négociation publique. Ces transformations ne font qu'accroître le sens d'insécurité, des inégalités internes grandissantes ainsi que l'importance accordée aux médias, à la consommation et surtout, aux lieux de consommation.

Par exemple, le déploiement dans le monde entier de grands centres commerciaux a provoqué des transformations irréversibles du territoire et a bouleversé les modes de consommation. Dans ces grands centres, les visiteurs auraient l'impression de se trouver dans une ville protégée et à l'abri de toute menace, dans une citadelle inexpugnable où tout danger extérieur est banni et toute agression environnementale écartée en contraste avec la ville en général, lieu à « insécurité croissante » évoqué cidessus. La surveillance, présente de manière massive, y est exercée par des systèmes technologiques ultrasophistiqués. En effet, le centre commercial, bien qu'il ne soit pas véritablement une ville, apparaît pourtant comme son double à la fois fictif et idéalisé : c'est la citadelle du «shopping" qui réduit la dimension urbaine à un espace de consommation et d'achats. Toutefois, elle n'a pas l'étendue de la vraie ville, elle n'en a pas les ouvertures, elle n'en a pas non plus les problèmes, ni les contradictions. Dans un certain sens, on peut avoir l'impression que les citadelles $d u$ shopping protègent l'individu de cette "société du risque » dont parle Ulrick Beck (2000), où les menaces sont engendrées par la société elle-même et réparties en son sein.

\section{Potentialités des villes}

Selon un autre point de vue, en dépit du contexte ci-dessus exposé, c'est dans les villes que l'on échange, que l'on se rencontre et que l'on bouge. C'est dans les villes, par opposition aux campagnes féodales, que sont nées les libertés modernes et l'idée d'égalité. C'est justement pour cette raison que la ville apparaît comme le lieu où l'être humain semble le mieux pouvoir respirer la liberté et trouver cet espace de mouvement qu'il recherche constamment (Bauman, 2002). Selon ce point de vue, il est possible à l'intérieur de la grande ville d'expérimenter, chaque jour, des situations nouvelles. La diversité des contacts et des confrontations possibles ainsi que leur caractère aléatoire permettraient d'entrer en relation avec des comportements, des goûts, des informations que l'on ne connaissait pas, ce qui accroît les possibilités 
d'apprentissage de nouvelles propositions culturelles et permet un échange éducatif, une écoute et une participation. nécessaire au développement de la pensée critique et constitue une stratégie appropriée pour relever les défis du XXIe siècle dans nos villes. Aborder la créativité, qui est un concept assez récent (né au XIXe siècle, mais davantage étudié au XXe siècle, selon Melucci, 1994), requiert avant tout de déblayer le terrain de toute équivoque possible et d'aller au-delà des stéréotypes. Selon un stéréotype, la créativité est un phénomène d'élite, une expression du génie solitaire (Montuori, 2003). Malheureusement, s'il se déploie isolément, le talent créatif n'atteint pas une masse critique et ne permet pas la mise en œuvre d'innovations. Il existe aussi une créativité de groupe (Montuori, 2003) qui s'exprime aussi bien dans les réseaux de recherche que sous de nombreuses formes artistiques (jazz, cinéma, par exemple). La créativité peut représenter un patrimoine collectif et non pas un don individuel, une créativité diffuse (Montuori, 2003) quand plusieurs facteurs sociaux, économiques et géographiques la rendent possible. Appliquée aux organisations, la créativité diffuse requiert l'émergence de nouvelles formes de relations entre les membres de l'organisation, qui soient non rigidement hiérarchiques, mais plutôt souples.

Lacte créatif n'est donc pas un acte solipsiste, mais afin qu'il soit le moteur d'une transformation effective, il doit être ouvert à la communication intersubjective. En effet, dans le processus créatif, l'individu y met du sien, il puise dans ses ressources internes et met en jeu sa sensibilité et sa façon originale de vivre et d'entendre la réalité urbaine. Cela débouche sur un processus et des résultats qui enrichissent non seulement la personne qui les réalise, mais aussi le monde qui l'entoure, car c'est la naissance effective de quelque chose de nouveau qu'on peut partager. Il s'agit de la créativité d'un homo civicus (Cesareo et Vaccarini, 2006) qui justement, grâce à son fort caractère, a des rapports fréquents et profonds d'interdépendance avec ses semblables 
et avec l'histoire collective. C'est une personne qui sait embrasser de larges espaces de temps, en faisant des choix de façon autonome et responsable (Cesareo et Vaccarini, 2006).

18 La créativité dont nous parlons n'est pas celle de nombreux manuels qui prétendent l'enseigner et la considèrent comme la clé du succès personnel. Elle n'est pas non plus l'outil de "marketing» qui associe la créativité à la compétitivité de l'entreprise capitaliste indépendamment du contexte et des jugements de valeur portés. Elle ressemble plutôt à l'action sociale responsable, définie par Cesareo et Vaccarini (2006) comme un jeu sérieux, c'est-à-dire une action créative ludique, mais aussi sérieuse, comme toute action relative à la vie collective.

Certes, il est vrai que la publicité vend une identité plus qu'un produit, mais c'est parce que le marketing exploite le désir croissant de se créer soi-même. Dans le monde entier, il existe aujourd'hui un désir majeur de satisfaction personnelle, d'autocréation individuelle. Mais il existe aussi une énorme explosion de créativité globale due aux interactions culturelles. Cette énorme explosion de créativité s'exprime "dans les aliments que nous mangeons, dans les livres que nous lisons, dans les films que nous regardons, dans les vêtements que nous portons, dans les types de soins que nous choisissons, dans les pensées que nous avons et dans les traditions spirituelles que nous adoptons » (Montuori, 2004, p. 37) et qui viennent désormais de tous les continents.

\section{Créativité et complexité}

20 Une éducation relative à l'environnement critique gagnerait à considérer ce phénomène de désir et de quête de créativité. Elle pourrait servir de guide à la créativité, notamment pour les raisons suivantes:

- La créativité, si elle est bien exercée, est essentiellement anticonformiste, antitotalitaire et antiautoritaire, le propre d'une société plurielle et décentralisée.

- D’un point de vue épistémologique, la créativité est liée à la complexité, à une vision créative et non pas mécanique de l'univers et de la société. En effet, penser de manière créative signifie reproduire le processus évolutif de la vie qui est lui-même créatif, vivre avec l'incertitude et accepter l'imprévisibilité, saisir de nouvelles connexions et interactions, apprécier les propriétés émergentes des choses. Les personnes créatives, par exemple, estiment la complexité (Monceri, 2004). En ce sens, la créativité s'oppose au réductionnisme et, en tant que capacité à formuler de nouvelles questions et inventer de nouvelles solutions, elle correspond au mode de pensée appelé « la découverte ou l'invention des problèmes » (traduction libre, Monceri, 2004).

21 Comme on peut le voir, on aborde ici un point fondamental de l'éducation relative à l'environnement: il est impossible d'affronter la question socio-environnementale contemporaine avec les mêmes schémas mentaux et la même épistémologie qui sont à la base de la crise écologique planétaire. Effectivement, il faudrait aborder la crise socio-écologique contemporaine dans une perspective de rupture des schémas mentaux précédents. Ce changement nécessite entre autres de la créativité. Il s'agit d'apprendre « à penser de manière différente, créative, pour nous permettre de voir au-delà des choses, au-delà de la séparation et de l'identité, au-delà des parties fragmentées, dans un tout créatif et vivant» (traduction libre, Low, 2004, p. 63). Du reste, ce sont bien les nombreux écologistes « radicaux » et les mouvements de la société civile de la seconde moitié du XXe siècle qui introduisent des éléments «hérétiques» et innovants de la 
pensée et qui produisent les premières transformations concrètes dans l'organisation des villes où, à côté des transformations provoquées par la mondialisation, émergent aussi des expériences alternatives intéressantes (Landry, 2000).

\section{Utilisation créative des choses}

L'un des points cruciaux pour une diminution de l'empreinte écologique de l'humanité, qui a triplé entre 1961 et 2006 (WWF, 2006) est notamment la "dématérialisation de l'économie " ", à savoir la réduction de la matière et de l'énergie qui alimentent actuellement l'insoutenable modèle de production et de consommation.

Comme on l'a déjà dit, les villes sont devenues (pour les classes les plus aisées, mais aussi pour les classes moins aisées qui ont tendance à les imiter) des centres de consommation aliénée, c'est pourquoi

[...] nous achetons des vêtements, de l'ameublement, des électroménagers, des téléphones mobiles, des voitures, non pas en fonction de réels besoins ou d'un plaisir effectif à les utiliser, mais uniquement dans le but d'acheter, de posséder et de montrer aux autres cette possession. [...] d'où l'autre danger, à savoir une consommation d'énergie non renouvelable et de matières premières essentielles (il suffit de penser aux hydrocarbures et au bois) sans fin, ce qui comporte de graves dangers liés à la destruction de l'environnement, à la pollution et, en dernière analyse, un risque terrible pour le développement durable de la planète. (Traduction libre, Inghilleri, 2003, p. 28)

La construction d'une solution alternative, vers de nouvelles réalités socio-écologiques, passe aussi par un rapport différent aux objets, qu'ils soient matériels, et dont la demande du marché semble aujourd'hui infinie (mais destinée à se heurter aux limites de la planète Terre), ou immatériels (les créations humaines constituées par des communautés, des idéologies, des institutions, des systèmes politiques, etc.). C'est la perspective de se soustraire à la fièvre de la consommation par une utilisation autonome et créative, autodéterminée et consciente des choses, fondée sur les relations, sur l'attention, sur la responsabilité globale (Inghilleri, 2003). Pour que la créativité puisse se déployer et devenir une force critique et transformatrice, il faut qu'elle soit promue et soutenue comme dimension non instrumentale, collective, visant à un avenir différent et meilleur, et que toute la ville devienne " éducative ", ce qui est tout à fait différent d'une ville «bien éduquée ».

[La ville éducative est] un lieu où les individus et les organisations sont encouragés à apprendre les dynamiques du lieu où ils vivent et la façon dont il change ; un lieu qui change la façon d'apprendre à comprendre les opportunités d'emploi dans le système formel et informel; un lieu où tous ses membres sont encouragés à apprendre ; enfin, et c'est peut-être le plus important, un lieu qui peut enseigner à changer les conditions de son apprentissage de manière démocratique. (Traduction libre, Landry, 2000, p. 267)

\section{Il s'avère donc de plus en plus nécessaire d'apprendre à}

[...] appliquer la créativité humaine à notre habileté à vivre dans un monde pluriel, pour créer des mondes à une époque où une compréhension du monde univoque et homogène n'est plus réalisable. Et cela sera nécessaire justement pour cette solidarité qui verra les êtres humains ensemble au lieu de les voir comme des fragments d'une opposition infinie. (Montuori, 2003, p. 48) 
alors d'intégrer les potentialités de la ville et une conception « collective » de la créativité en éducation relative à l'environnement pour transformer les villes et nous transformer nous-mêmes.

\section{La pensée critique et la vie dans les villes}

Du reste, c'est l'appel éthique, épistémologique et culturel à une approche critique et holistique de la réalité, propre à l'éducation relative à l'environnement, qui nous protège du risque d'un "savoir-marchandise » sur la ville. En effet, les connaissances ont tendance à se stabiliser dans un rapport avantageux sur le plan personnel selon l'utilité de notre point de vue, dans le sens où le rôle des autres dans notre vie est essentiellement considéré comme un moyen pour atteindre nos objectifs. "Alors que l'individu gagne, d'une part, un certain degré de liberté ou d'émancipation des contrôles personnels et émotifs d'une partie du groupe des intimes; de l'autre, il perd sa spontanéité, sa morale et son sens de participation » (traduction libre, Wirth, 1998, p.60) dérivant du fait qu'il vit dans une société très organisée. Par ailleurs, le développement d'une pensée critique en éducation relative à l'environnement et d'une "conscientisation" des habitants peut représenter une clé de voûte capable de transformer la situation actuelle de désagrégation sociale dans nos métropoles. Il s'agit d'une conscientisation non seulement comme prise de conscience, mais aussi, selon Paulo Freire (1997), comme action profonde de l'être humain sur la réalité sociale. « La conscience critique est la perception des choses et des faits tels qu'ils surviennent dans l'existence empirique, dans leurs rapports logiques et circonstanciels " (traduction libre, Freire, 1997, p. 153). D'après cet auteur, «les rapports humains renferment un ensemble de notions telles que le pluralisme, la capacité de jugement critique, la créativité et la dimension temporelle» (traduction libre, Freire, 1997, p. 170). L'être humain, contrairement aux animaux, est un sujet de rapports et pas seulement de contacts. En plus d'être dans le monde, il est avec le monde et donc ouvert à la réalité. Quand l'individu comprend la réalité ou les rapports qui relient un fait à un autre, son action devient naturellement critique, c'est-à-dire qu'elle devient réflexion et non pas réflexe, comme cela se produit dans la sphère des simples contacts (Freire, 1997). À travers ses rapports avec la réalité, fruit de sa présence dans le monde et avec le monde, au moyen de ses gestes de création, recréation et décision, l'être humain rend l'histoire dynamique; il l'humanise en y ajoutant quelque chose que lui-même a créé (Freire, 1997).

Comme nous avons tenté de l'illustrer, le caractère fragmenté et la dimension utilitariste des rapports interpersonnels dans la ville trouvent leur expression dans les institutions et dans la prolifération de fonctions spécialisées. Dans une communauté trop grande pour que les individus puissent se connaître de manière très intime et être réunis dans un unique lieu, il est devenu nécessaire de communiquer grâce à des moyens indirects (médias, technologies de l'information et des communications) et d'articuler les intérêts individuels à travers un processus de délégation à des représentants (d'institutions, d'associations, d'organisations du loisir, etc.). On se demande alors s'il est possible de développer une pensée critique autonome dans un système urbain écrasé par le poids des médias et par leur pouvoir de manipulation. L'éducation est un moyen, peut-être le seul moyen, pour y arriver.

Éducation relative à l'environnement, Volume 7 | 2008 
29 C'est dans ces difficultés et ces menaces que se situe le rôle fondamental de l'éducation relative à l'environnement : travailler avec les personnes, former les citoyens afin qu'ils développent des liens sociaux et qu'ils soient aptes à s'interroger sur ces derniers et que, grâce à eux, ils accueillent les altérités, qu'elles proviennent de la communauté urbaine ou de l'extérieur. Ce n'est qu'à travers le dialogue éducatif que l'on construit ce rapport indispensable pour son avenir dans et avec le monde (Freire, 1997, p. 170). Être dans le monde et avec le monde signifie non seulement d'apprendre à lire la réalité, mais aussi de se proposer de la modifier. Un sujet est capable de se transformer dans la mesure où il est capable de provoquer un changement (Freire, 1997). Cette façon d'agir permet à l'individu de devenir l'agent de sa réhabilitation et de se placer dans une position critique par rapport au contexte. Entre autres, être dans le monde et avec le monde est aussi une façon de bâtir une vraie démocratie en tant que processus complet, conscient, d'appartenance, de responsabilité, mais aussi de pouvoir dans la vie de la communauté. "L'éducation problématisante stimule la réflexion et l'action de l'être humain sur la réalité en répondant à sa vocation qui est authentique uniquement dans la mesure où elle vise à réaliser une transformation " (traduction libre, Freire, 2002, p. 65). Dans cette perspective, le citoyen est capable de provoquer des changements dans l'ordre existant et de contribuer à la création d'une meilleure qualité de la vie.

Par ailleurs, la réflexion doit être conçue comme étant orientée vers l'action (Kemmis, 1982). De plus, Beck et ses collaborateurs (1999) soutiennent la nécessité, pour la société moderne, de devenir une société réflexive et d'accroître la discussion publique sur les problèmes à aborder. Il s'agit donc d'étendre la démocratie du dialogue et de la confrontation, basée sur la confiance active des acteurs sociaux, à divers niveaux: relations interpersonnelles, société civile, institutions nationales et internationales. Cet aspect devient la condition nécessaire pour une action en réseau et par des réseaux. Dans cette perspective, l'acteur n'est jamais un seul protagoniste, c'est un acteur pluriel parce qu'il est constitué d'un réseau de sujets agissant sur la base d'un projet de responsabilité distribuée. Il arrive donc que les citoyens impliqués dans un processus de formation à la pensée critique constituent une "communauté critique de chercheurs » (Carr et Kemmis, 1986, p. 40). La formation à la pensée critique et créative dans un cadre de participation des citoyens à la vie démocratique permet un échange d'expériences, de points de vue, de connaissances, d'idées. C'est un processus d'apprentissage collectif qui ressemble à une recherche bien structurée et qui nécessite organisation et ressources.

31 La transformation urbaine tant souhaitée ne pourra donc pas être atteinte par l'individu à lui seul. Elle devra être le résultat d'un processus qui se réalise dans le rapport entre les humains par l'intermédiaire du monde (Beck, 2003). Les processus décisionnels doivent ainsi s'ouvrir au corps social et encourager tous les citoyens à abandonner leurs habitudes, les lieux communs, leurs préjugés pour retrouver la capacité d'inventer, de jouer, de rêver. Parce qu'elle est une entité complexe, pour être écologique, la ville requiert le développement de la créativité et à l'inverse, pour être créative, elle doit devenir écologique.

32 En conclusion, l'approche critique en éducation relative à l'environnement permet de cibler les inégalités, le gaspillage de ressources, l'arrogance du domaine humain sur la nature, mais son apport réel à un développement urbain contemporain approprié mérite d'être davantage appuyé. Il y a nécessité de développer la recherche en éducation relative à l'environnement en milieu urbain et les expériences relatives à 
l'environnement urbain ${ }^{1}$. La clé d'un apport majeur de l'ERE à des villes viables et écologiques est peut-être le développement de la créativité des habitants de la ville. Entre éducation relative à l'environnement et créativité, il y a en effet un lien très fort, parce que l'ERE s'intéresse à une citoyenneté active, à un changement de la pensée et à la recherche d'idées nouvelles quant au modèle contemporain de production et de consommation.

\section{BIBLIOGRAPHIE}

Bauman, Z. (2002). Modernità liquida. Bari-Roma : Laterza.

Bauman, Z. (2005). Fiducia e paura nella città. Milano : Bruno Mondadori.

Beck, U. (2000). I rischi della libertà. L'individuo nell'epoca della globalizzazione. Bologna : Il Mulino.

Beck, U. (2003). La società cosmopolita. Prospettive dell'epoca postnazionale. Bologna : Il Mulino.

Beck, U., Giddens, A. et Lash, S. (1999). Modernizzazione riflessiva. Politica, tradizione ed estetica nell'ordine sociale della modernità. Trieste : Asterios.

Caritas Italiana. (2007). La città abbandonata. Bologna : Il Mulino.

Carr, W. et Kemmis, S. (1986). Becoming critical : education knowledge and action research. Londres : Falmer Press.

Cesareo, V. (2001). Globalizzazione e contesti locali. Milano : FrancoAngeli.

Cesareo, V. et Vaccarini, I. (2006). La libertà responsabile. Soggettività e mutamento sociale. Milano : Vita e Pensiero.

Freire, P. (1997). Educazione come pratica di libertà. Milano : Mondadori.

Freire, P. (2002). La pedagogia degli oppressi. Torino : EGA Editore.

Hannerz, U. (2001). Esplorare la città. Antropologia della vita urbana.Bologna : Il Mulino.

Inghilleri, P. (2003). La « buona vita ». Per l'uso creativo degli oggetti nella società dell'abbondanza.

Milano : Guerini e Associati.

Kemmis, S. (1982). The action research planner. Geelong : Deakin University Press.

Landry, C. (2000). The Creative City. A Toolkit for Urban Innovations.Londres : Earthscan.

Low, A. (2004). Il pensiero creativo. Élites, 4, 55-64.

Mazzette, A. (2003). La città che cambia. Milano : FrancoAngeli.

Mazzette, A. (2006). L'urbanità delle donne. Creare, faticare, governare e altro. Milano : FrancoAngeli.

Mela, A. (2006). Sociologia delle città. Roma : Carocci.

Melucci, A. (1994). Creatività : miti, discorsi, processi.Milano : Feltrinelli.

Merton, R.K. et Barber, E. (2002). Viaggi e avventure della serendipity. Saggio di semantica sociologica e sociologia della scienza. Bologna : Il Mulino. 
Monceri, F. (2004). Il pensiero dissonante. Sulla creatività del filosofo. Élites, 4, 38-49.

Montuori, A. (2003). La complessità della creatività e la creatività della complessità. Élites, 3, $42-48$.

Montuori, A. (2004). Ricreare la creatività. Élites, 4, 32-37.

Nancy, J.-L. (2002). La città lontana. Verona : Ombre Corte.

Sassen, S. (2003). Le città nell'economia globale. Bologna : Il Mulino.

Sennett, R. (2006). La cultura del nuovo capitalismo.Bologna : Il Mulino.

UNDESA, United Nations Department of Economic and Social Affairs - Population Division.

(2008a). World Urbanization Prospects. The 2007 Revision. New York : Nations Unies.

UNDESA, United Nations Department of Economic and Social Affairs - Population Division.

(2008b). United Nations expert group meeting on population distribution, urbanization, internal migration and development. New York : Nations Unies.

UNFPA, Fonds des Nations Unies pour la population. (2007). État de la population mondiale 2007. Libérer le potentiel de la croissance urbaine. New York : UNFPA.

Wirth, L. (1998). L'urbanesimo come modo di vita. Roma : Armando Editore (édition originale, 1938).

WWF - World Wildlife Fund. (2006). Living Planet Report 2006. Gland : WWF International.

\section{NOTES}

1. Un état des lieux de l'éducation relative à l'environnement en milieu urbain et un panorama d'outils et de ressources sont téléchargeables à partir du site Internet www.urban-education.org (aussi disponible sur CD-Rom) dans le cadre du projet "Urbanet », sous la direction de Mario Salomone.

\section{AUTEURS}

\section{MARIO SALOMONE}

Sociologue de l'environnement et professeur en éducation relative à l'environnement à l'Université de Bergame (Italie) et directeur et fondateur du mensuel italien Eco l'educazione sostenibile et de la revue scientifique Culture della sostenibilità, il a organisé le troisième Congrès mondial de l'éducation relative à l'environnement (WEEC, octobre 2005) et coordonne le réseau international qui y est associé.

\section{MARCELLA MESSINA}

Doctorante en anthropologie et en épistémologie de la complexité à l'Université de Bergame (Italie), elle est assistante en éducation relative à l'environnement à la même université, collaboratrice du mensuel .Eco et secrétaire de rédaction de la revue scientifique Culture della 
sostenibilità. Elle travaille aussi à titre de consultante psycho-pédagogique et de coordonnatrice en éducation. 